\title{
UAS Integration into the NAS: An Examination of Baseline Compliance in the Current Airspace System
}

\author{
Lisa Fern, San Jose State University \\ Caitlin A. Kenny, San Jose State University \\ Robert J. Shively, U.S. Army AMRDEC \\ Walter Johnson, NASA Ames Research Center
}

\begin{abstract}
As a result of the FAA Modernization and Reform Act of 2012, Unmanned Aerial Systems (UAS) are expected to be integrated into the National Airspace System (NAS) by 2015. Several human factors challenges need to be addressed before UAS can safely and routinely fly in the NAS with manned aircraft. Perhaps the most significant challenge is for the UAS to be non-disruptive to the air traffic management system. Another human factors challenge is how to provide UAS pilots with intuitive traffic information in order to support situation awareness (SA) of their airspace environment as well as a see-and-avoid capability comparable to manned aircraft so that a UAS pilot could safely maneuver the aircraft to maintain separation and collision avoidance if necessary. A simulation experiment was conducted to examine baseline compliance of UAS operations in the current airspace system. Researchers also examined the effects of introducing a Cockpit Situation Display (CSD) into a UAS Ground Control Station (GCS) on UAS pilot performance, workload and situation awareness while flying in a positively controlled sector. Pilots were tasked with conducting a highway patrol police mission with a Medium Altitude Long Endurance (MALE) UAS in L.A. Center airspace with two mission objectives: 1) to reroute the UAS when issued new instructions from their commander, and 2) to communicate with Air Traffic Control (ATC) to negotiate flight plan changes and respond to vectoring and altitude change instructions. Objective aircraft separation data, workload ratings, SA data, and subjective ratings regarding UAS operations in the NAS were collected. Results indicate that UAS pilots were able to comply appropriately with ATC instructions. In addition, the introduction of the CSD improved pilot SA and reduced workload associated with UAS and ATC interactions.
\end{abstract}

\section{INTRODUCTION}

Public demand to fly Unmanned Aerial Systems (UAS) in the U.S. National Airspace System (NAS) came to a head this year when the Government signed into law the FAA Modernization and Reform Act of 2012, which requires a plan for full integration of UAS into the NAS by 2015 (Hinton, 2012). Among the provisions of the bill, the Federal Aviation Administration (FAA) will be required to study UAS human factors and causes of incidents. Several human factors challenges need to be addressed before UAS can safely and routinely fly in the NAS. Perhaps the most significant challenge is for the UAS to be non-disruptive to the air traffic system, despite a variety of unique performance and operational characteristics. This in turn highlights two main human factors issues unique to UAS: the lack of in-situ sensory input and feedback (aural, visual and vestibular cues) for the pilot, and the lack of uniform and standardized communication, navigation and traffic information equipment (Fern, Shively, Johnson, Trujillo, Pestana \& Hobbs, 2011).

Current unmanned systems vary widely in their implementation of these systems, and to date no UAS includes a traffic display of information in the Ground Control Station (GCS), and no standards for UAS traffic displays exist. However, through Directive 7610.4J (2004), the FAA has mandated that UAS (referred to as "ROA" in the directive) operations require "the proponent to provide an equivalent level of safety, comparable to see-and-avoid requirements for manned aircraft." There is substantial debate whether this requirement could be met through the use of the Traffic Alert and Collision Avoidance System (TCAS) on UAS. TCAS is a family of airborne devices that function independently of ground-based sense and avoid and is mandated on all aircraft with 10 seats or more (FAA, 2010). All TCAS systems provide some degree of collision threat alerting in addition to a traffic display, which can assist a pilot in visual acquisition of intruder aircraft. Given the lack of a means to visually acquire and monitor aircraft operating near a UAS, the TCAS display has been proposed as a means to provide the awareness of the traffic environment to at least partially meet the "comparable see-and-avoid" requirement (a fully integrated solution could be expected to include a sense-andavoid system).

Unfortunately, TCAS has generally been discouraged by the FAA for use on UAS. Among the reasons for this is that maneuvering an aircraft solely on the basis of traffic advisory symbology presented on the TCAS display was explicitly prohibited as part of its operational approval (FAA, 2011a). TCAS is intended to serve as a backup to the primary means of collision avoidance: visual collision avoidance (through see-and-avoid), application of right-of-way rules, and air traffic separation service provided by Air Traffic Control (ATC). It was not designed to be relied on as a primary source of collision avoidance. Additionally, in a study conducted by the FAA to identify and evaluate potential uses of TCAS on UAS (2011b), it was concluded that installation and use of TCAS on UAS should not be authorized for horizontal and vertical maneuvers to maintain self separation, nor as a means of providing situation awareness (SA) due to inaccurate and incomplete traffic information provided by the TCAS display. 
In positively controlled airspace, UAS can maintain separation assurance with other traffic through separation services provided by ATC, which do not rely on a pilot's ability to see-and-avoid. However, there still remains a need to provide UAS pilots with necessary traffic information in an integrated and intuitive fashion in order to meet the comparable see-and-avoid requirement. This is needed in order to support safe flight in other classes of airspace where some or all traffic rely on visual flight rules (VFR) to self separate and ATC separation services may not be provided, or in rare situations where ATC separation services fail. In such instances, pilots need sufficient SA in order to maneuver safely to avoid losses of separation and/or collisions with other aircraft. In addition, while dependence on air traffic separation services can substantially reduce the risk of UAS collisions, the integration of UAS into an already at-capacity air transportation system could potentially increase ATC cognitive and physical workload to unacceptable levels, especially if substantial special handling or procedures are required due to unique UAS characteristics, such as the potential for lost link events. Thus, attention to ATC workload is critical to any potential solution for UAS integration.

This experiment investigated two main objectives. The first was to examine baseline conditions for a UAS operating in current generation, positively controlled airspace alongside manned aircraft. This baseline included establishing whether the UAS could operate with a shared Navigational Aid (NavAid) database in the GCS, whether the UAS pilot could follow current instrument flight rules (IFR) procedures and comply appropriately with ATC instructions, and whether a controller could maintain acceptable levels of workload and safety when a UAS was in their sector. The second objective was to examine the effects of introducing a basic traffic display into a UAS ground control station on UAS pilots' workload, SA, and ability to maintain separation assurance with other aircraft while operating under separation services provided by ATC.

Previous research examining the effects of introducing graphical traffic and airspace displays into a UAS GCS for military operations have found improvements in workload, SA and pilot ability to maintain separation from other aircraft (Fern, Flaherty, Shively \& Turpin, 2011; Fern \& Shively, 2011). Due to the separation services provided by ATC, it was hypothesized that pilot ability to maintain separation would not be affected by the presence of a display. However, pilot workload was expected to decrease, and SA was expected to increase, with the added information that the display provided. ATC workload and subjective ratings about UAS operations in civil airspace were also collected and reported.

\section{METHOD}

\section{Participants}

Twelve pilots were recruited to participate as UAS pilots in this study. All were males (averaged 29.25 years) with an average of 1981.75 flight hours. Total flight hours ranged from 265 to 5000 hours. Participants were required to hold at minimum, a Private Pilot Certificate and an Instrument Rating.
No pilots reported military experience, though one reported UAS flight experience. Eligibility was limited to participants who had normal or corrected to normal vision and were under 40 years old. Two retired air traffic controllers and two pseudo-pilot confederates were also recruited to participate in the study. Participants were compensated for their participation in the study.

\section{Simulation Environment}

Participants interacted with the simulation software using desktop PCs and standard keyboard and mouse inputs. The UAS pilot participants were situated at a UAS Ground Control Station (GCS) that consisted of the Multiple-UAS Simulator (MUSIM) and the Cockpit Situation Display (CSD) (Figure 1). A participant controller and confederate pilot each used a separate mode of the Multi-Aircraft Control System (MACS; see Prevot, 2002) software to manage and control AC in the simulation.

MUSIM is a Linux-based UAS GCS simulation environment (see Fern \& Shively, 2009). The current simulation configuration of MUSIM differed only slightly in that it utilized a 1:1 operator to vehicle interface with a generic fixed wing flight control model input with generic Medium Altitude Long Endurance (MALE) UAS parameters. Ownship airspeed was fixed at $110 \mathrm{kts}$ for the entire experiment. MUSIM was separated into three Graphical User Interfaces (GUIs): a map display indicated the position and flight plan of the UAS, and included NavAid waypoints to be used for all flight plans; a multi-function display (MFD) contained the UAS primary flight display and provided mission messages; and a mission timer.

The CSD is an interactive 3D volumetric display designed to provide pilots with awareness of their surroundings (see Granada, Dao, Wong, Johnson \& Battiste, 2005). For this experiment, it was used to display traffic and trajectory information in its basic 2D planar view. The CSD had an ownship-centric view of surrounding airspace and provided participants with trajectory information of the surrounding aircraft. Participants were able to adjust the horizontal viewing distance from 10-640 $\mathrm{nm}$ and display aircraft trajectories. No other manipulations were allowed on the CSD during this experiment.

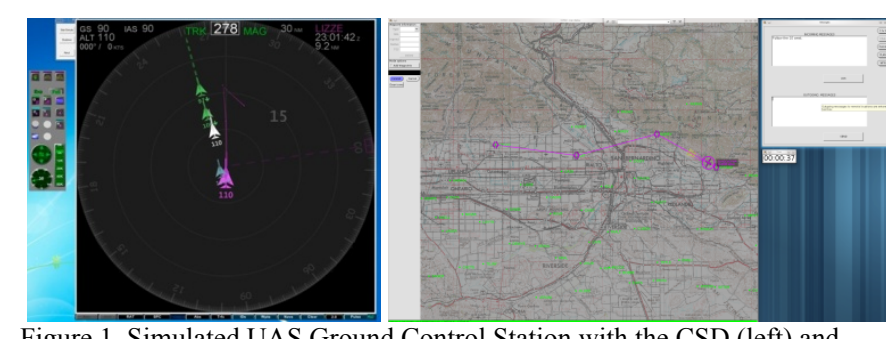

Figure 1. Simulated UAS Ground Control Station with the CSD (left) and MUSIM (right).

MACS is an emulation and simulation program that provides a small to large scale airspace environment. In the current experiment, MACS included displays for the confederate pilot and the display system replacement (DSR) scope used by the controller. The low altitude center sector 
owned by the controller was from $13,000-24,000 \mathrm{ft}$. and the sector boundary was highlighted in comparison to the surrounding sectors. The same NavAid waypoints available to the UAS pilot were also displayed in order to provide a common reference to support ATC-pilot communications. Control instructions given by the controller to all aircraft were delivered via voice communication over a simulated radio frequency to either the confederate pilot or the UAS pilot.

\section{Experimental Design}

A within-subjects, repeated measures design was used to study operator performance, workload, and SA measures while flying a highway patrol police mission using a MALE UAS in high density L.A. Center area airspace. Two traffic display conditions (no display, CSD present) were compared against two different traffic densities (low, high). CSD presentation and traffic density were counterbalanced across participants and scenarios.

Traffic Display. The CSD was introduced in half of the scenarios to provide pilots with traffic information. Operators were able to adjust the horizontal viewing distance from 10$640 \mathrm{~nm}$ and display aircraft trajectories to assist them in flying their highway patrol missions. Aircraft were color-coded based upon altitude relative to the UAS: aircraft $500 \mathrm{ft}$ or more below the UAS were green, aircraft between $500 \mathrm{ft}$ above or below the UAS were white, and aircraft $500 \mathrm{ft}$ or more above the UAS were blue. To bring up aircraft trajectories, operators right clicked on the desired aircraft. In the baseline condition with no traffic display, which mimics current UAS GCS, the CSD monitor was turned off.

Traffic Density. Two levels of traffic density, high and low, were used. In the high traffic density condition (half of the scenarios) there were between 12 and 16 aircraft in the sector, while in the low traffic density condition there were between nine and 12 aircraft in the sector. The sector densities were vetted with subject matter experts during development.

Missions. One training and four experimental scenarios were developed for this experiment. The training scenario was five minutes long and provided flight plan editing practice for the pilots. Experimental scenarios were 30 minutes long and included manned IFR aircraft managed by a confederate pseudo-pilot, with a single controller providing separation services to all aircraft. All scenarios began with a preprogrammed flight plan and included five mission messages from a commander that required operator attention and rerouting throughout the mission. The timing of the reroute messages and flight paths flown differed between scenarios as a means to reduce predictability.

Mission Objectives. Participants were instructed to fly a highway patrol police mission L.A. Center airspace with two mission objectives: 1) to reroute the UAS when issued new instructions from their commander, and 2) to communicate with ATC to negotiate flight plan changes and respond to vectoring and altitude change instructions. The first objective required operators to fly the initial pre-programmed mission flight plan while monitoring the MFD for messages that contained instructions from their commander. Upon receiving a reroute message, pilots created a new flight plan that followed the message instructions as closely as possible. The second objective required operators to maintain radio contact with ATC while flying their mission, request clearance for new flight plans, and respond to instruction to vector or change the altitude of the UAS to avoid other traffic.

\section{Procedure}

Participants were required to complete an informed consent for minimal risk form and a demographic survey intended to elicit information regarding their flight experience.

Training Sessions. After completing paperwork, pilots were given a short briefing introducing MUSIM and mission objectives. After the initial briefing, participants completed a training scenario to familiarize themselves with MUSIM and how to make flight plan changes. An additional briefing was provided before the CSD experimental block detailing how to use the CSD.

Experimental Sessions. The experimental sessions were blocked by traffic display (no display vs. CSD present). Participants completed four experimental missions during the simulation. Before each experimental block, participants were given a practice mission to familiarize themselves with the display condition. After each mission, participants completed a NASA TLX (Hart \& Staveland, 1988) to measure workload. Following each experimental block, participants completed a questionnaire to measure SA. At the end of the experimental session, operators completed a post-simulation questionnaire.

\section{MEASURES}

\section{Objective Performance}

Horizontal and vertical separation distances between the ownship and all other aircraft were collected. Conflicts that occurred within 750 feet vertical separation of ownship (regardless of horizontal separation), and lasting longer than 30 seconds were analyzed.

Minimum Horizontal and Vertical Distance. The minimum horizontal distance from the ownship to the intruder aircraft during a conflict event was measured in nautical miles (nm). The minimum vertical distance was measured in feet (ft).

Number of Losses of Separation. The number of loss of separation (LOS) events that occurred with the UAS ownship were collected. LOS was defined as a vertical separation of less than $750 \mathrm{ft}$ and a horizontal separation of less than $5 \mathrm{~nm}$.

\section{Workload}

NASA TLX. A NASA TLX was administered to both the pilot and the controller after each trial. Participants rated six dimensions of workload (mental, physical, temporal, effort, frustration, and performance) on a seven-point scale. An overall workload rating was also collected.

Pilot/ATC Interactions. In addition to the NASA-TLX, pilots and controllers each rated their overall workload level in interacting with each other on a seven-point Likert scale $(1=$ low, 7 = high). 


\section{Situation Awareness}

Following each display block, pilots were given six statements to assess their own situation awareness (SA) on an eight-point Likert scale, with 0 indicating low SA and 7 indicating high SA.

\section{RESULTS}

Conflict and pilot workload data were analyzed using a 2 (traffic display: no display, CSD present) X 2 (traffic density: high, low) repeated measures analysis of variance (ANOVA). No significant interactions between display and traffic density were found. Pilot SA ratings were analyzed using a one-way repeated measures ANOVA with traffic display as the main factor. Post hoc analyses utilized Bonferonni pairwise comparisons. Descriptive statistics only are provided for the ATC workload ratings which were averaged across trials.

\section{Objective Performance}

Minimum Horizontal and Vertical Distance. There were effects of display or density on minimum horizontal or vertical distances. The average minimum horizontal $(M=22.21 ; S E=$ $1.75)$ and vertical $(M=10.57 ; S E=4.82)$ distances with no display were not significantly different from the average minimum horizontal $(M=22.63 ; S E=1.93)$ and vertical $(M=$ 10.60; $S E=8.67)$ distances with the CSD present, $p>.05$.

Number of Losses of Separation. There was not a main effect of display on the number of loss of separation events, $F(1,11)=.0165, p>.05$. However, there was a main effect of traffic density on the number of losses of separation. The average number of losses of separation were significantly higher in the high density condition $(M=.072 ; S D=.29)$ compared to the low density condition $(M=.046 ; S D=.23)$, $F(1,11)=7.470, p<.05$.

\section{Workload}

Pilot TLX Workload Ratings. There was no main effect of display on NASA TLX workload ratings, however, the frustration dimension of workload trended toward significance. Average ratings of frustration appeared to be higher when no display was present $(M=2.08 ; S E=.40)$ than when operators were able to view the CSD $(M=1.67 ; S E=$ $.22), F(1,11)=3.873, p=.075$. There was no effect of traffic density on pilot workload ratings.

ATC TLX Workload Ratings. Mean workload ratings across the display conditions appeared flat. However, mean ratings of workload in the high traffic density condition appeared to be higher compared to the average workload ratings in the low density condition on the mental (6.5 vs. 5.8) and temporal (5.1 vs. 4.6) dimensions of workload, as well as overall workload (6.4 vs. 5.7).

Pilot/ATC Interactions. Pilots reported significantly higher workload with ATC interactions in the no display condition $(M=2.4 ; S E=0.40)$ compared to the CSD condition $(M=1.8 ; S E=.26), F(1,11)=6.494, p<.05$. Controllers also appeared to report higher workload levels when communicating with the UAS pilots when no display was present $(M=3.1 ; S E=.32)$ than when the CSD was present $(M=2.7 ; S E=.24)$. Pilot - controller interaction workload ratings are shown in Figure 2.

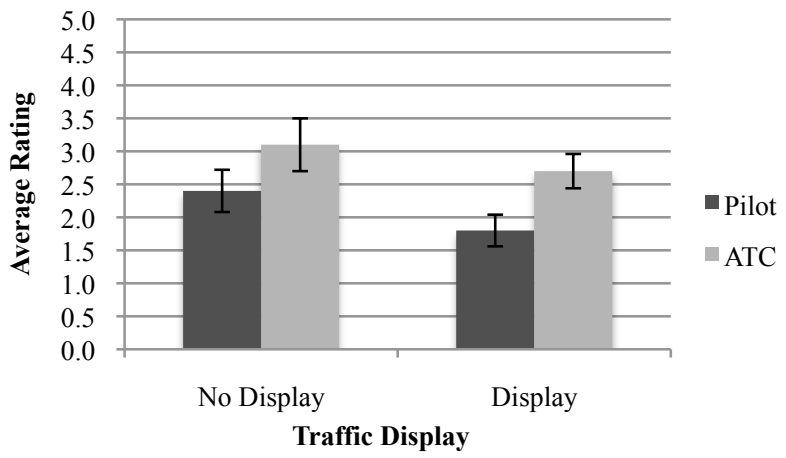

Figure 2. Pilot and controller interaction workload ratings.

\section{Situation Awareness}

Pilot self-ratings of SA were significantly higher with the CSD present compared to no display on five of the six SA questions. The means for the six SA statements are shown in Table 2.

\begin{tabular}{|c|c|c|c|c|}
\hline $\begin{array}{l}\text { Situation Awareness } \\
\text { Statement }\end{array}$ & $\begin{array}{l}\text { No Display } \\
\text { Mean Rating }\end{array}$ & $\begin{array}{l}\text { CSD Mean } \\
\text { Rating }\end{array}$ & $F(1,11)$ & $p$ \\
\hline $\begin{array}{l}\text { I was aware of the locations } \\
\text { of surrounding traffic }\end{array}$ & 0.9 & 5.4 & 68.538 & $<.001$ \\
\hline $\begin{array}{l}\text { I was confident in my } \\
\text { assessment of the traffic } \\
\text { situation }\end{array}$ & 1.3 & 5.9 & 40.931 & $<.001$ \\
\hline $\begin{array}{l}\text { I was aware of traffic } \\
\text { conflicts developing }\end{array}$ & 0.8 & 4.3 & 24.134 & $<.001$ \\
\hline $\begin{array}{l}\text { My SA was sufficient and } \\
\text { effective }\end{array}$ & 3.1 & 5.0 & 9.913 & $<.01$ \\
\hline $\begin{array}{l}\text { I had the airspace information } \\
\text { that I needed to complete } \\
\text { mission reroutes }\end{array}$ & 2.8 & 4.5 & 8.045 & $<.05$ \\
\hline $\begin{array}{l}\text { I was confident in my } \\
\text { responses to mission and ATC } \\
\text { requirements }\end{array}$ & 5.3 & 5.8 & 3.313 & $>.05$ \\
\hline
\end{tabular}

\section{DISCUSSION}

The preliminary results of this experiment indicate promising results with respect to the integration of UAS into the NAS. In post simulation questionnaires, the controller participants indicated that they felt that, compared to pilots of manned aircraft, the UAS pilots were able to comply immediately and appropriately to their instructions. They also felt that the UAS pilots had sufficient knowledge of the airspace and procedures, as well as an appropriate navigational database, to communicate with and respond appropriately to ATC. On average, the controllers reported that they used special handling procedures for the UAS only $0-25 \%$ of the time and utilized techniques that they would normally use with other special operations aircraft, such as giving the aircraft priority and vectoring or changing the altitude of conflicting aircraft. Overall, controllers felt that their workload with the 
addition of the UAS in their sector was "somewhat higher" than when managing a sector with only manned aircraft, and that meeting separation and flow requirements was "slightly more difficult."

The preliminary results also indicate some potential benefits to both pilots and controllers when the CSD is provided to UAS operating in positively controlled airspace. Although there were no reported differences in workload between the two display conditions, frustration appeared to decrease slightly when provided with information about the traffic environment. In addition, both pilots and controllers indicated lower workload levels when interacting with each other when the CSD was present. This is likely due to the UAS pilot's ability to judge the workload level of the controller and time his communications and requests for new flight plans accordingly. Controller workload ratings on all dimensions appeared to be higher on average than pilot ratings, supporting the notion that in positively controlled airspace the controllers shoulder the bulk of the workload in maintaining separation assurance and collision avoidance.

The largest benefit offered by the introduction of the CSD into the UAS GCS was on pilot SA. Pilots overwhelmingly judged their own SA as significantly higher when the CSD was present. However, self-reported measures of SA face certain limitations and may reflect a participant's confidence about their SA and their perceived performance more than their actual SA (Endseley, 1995). In this study, the presence of the traffic display may have increased their confidence of their SA, and therefore their SA ratings, simply because it has the appearance of providing more information. In addition, subjects simply may not be aware of critical information that they are missing. The addition of different measures of SA, such as objective performance measures, could be used to supplement the self-rating measures and give a more comprehensive evaluation of the effects of various displays on pilot SA.

While SA and some workload benefits were evident when UAS pilots were provided with the CSD, use of a traffic display in a UAS ground control station did not affect separation performance when operating in positively controlled airspace since the ATC carries the majority of the workload burden for maintaining safe separation between the UAS and other manned aircraft. Although losses of separation did occur infrequently (5-8\%), this data should be interpreted cautiously as UAS mission routes were specifically selected to be in conflict with traffic flows. In fact, the average minimum horizontal distance during reported conflicts (when vertical distance was $750 \mathrm{ft}$ or less) was $22 \mathrm{~nm}$, well within the separation boundary for civil aircraft.

Finally, varying traffic density had a predictable effect on ATC workload and loss of separation events, providing further evidence of the critical role of ATC in maintaining separation assurance in positively controlled airspace.

\section{Conclusion}

Overall, this simulation experiment yielded positive results for safe integration of UAS into the NAS. The controller workload ratings indicated acceptable levels of workload with the introduction of the UAS into their sector, and post simulation subjective responses revealed appropriate and timely compliance by the UAS pilot. However, the effect of UAS integration into civil airspace on ATC workload and ability to maintain traffic separation and flow requirements, particularly in dense airspace, deserves further research with larger sample sizes.

The presence of a traffic display is more likely to affect separation performance when there is a need for the pilot to maintain separation assurance and collision avoidance because ATC services are either not provided (i.e. different classes of airspace) or fail, or if pilots become responsible for self separation through new roles and responsibilities that could be introduced in the Nextt Generation (NextGen) environment. Further research looking into these potential situations is required in order to determine the benefits and requirements for presenting traffic information in a UAS GCS.

\section{REFERENCES}

Endsley, M. R. (1995). Toward a theory of situation awareness in dynamic systems. Human Factors, 37(1), 32-64.

Federal Aviation Administration. (2011a) Air Carrier Operational Approval and Use of TCAS II. Advisory Circular 120 -55C, Washington, DC, FAA.

Federal Aviation Administration. (2011b). Evaluation of candidate functions for traffic alert and collision avoidance system ii (TCAS II) on unmanned aircraft system (UAS) (FAA/AFS-407). Washington, DC: FAA Unmanned Aircraft Program Office.

Federal Aviation Administration (2004). Order 7610.4K special military operations (Distribution: ZAT-761; ZAT-464). Washington, DC: FAA.

Federal Aviation Administration (2010, August 4). TCAS home page. Retrieved from http://adsb.tc.faa.gov/TCAS.htm

Fern, L., Flaherty, S. R., Shively, R. J., \& Turpin, T. (2011). Airspace deconfliction for UAS operations. Proceedings of the 16th International Symposium on Aviation Psychology, Dayton, OH, May 3-5.

Fern, L., \& Shively, R. J. (2011). Designing airspace displays to support rapid immersion for UAS handoffs. Proceedings of the Human Factors and Ergonomics Society 55th Annual Meeting, Las Vegas, NV, September 19-23.

Fern, L., \& Shively, R. J. (2009). A comparison of varying levels of automation on the supervisory control of multiple UASs. In Proceedings of AUVSI's Unmanned Systems North America 2009, Washington, D.C..

Fern, L., Shively, R. J., Johnson, W., Trujillo, A., Pestana, M., \& Hobbs, A. (2011). Human factors challenges facing UAS integration into the NAS. Proceedings of the 16th International Symposium on Aviation Psychology, Dayton, OH, May 3-5.

Granada, S., Dao, A. Q., Wong, D., Johnson, W. W., \& Battiste, V. (2005) Development and integration of a human-centered volumetric cockpit display for distributed air-ground operations. In Proceedings of the 12th International Symposium on Aviation Psychology, Oklahoma City, OK.

Hart, S., \& Staveland, L. (1988). Development of NASA TLX (task load index): Results of empirical and theoretical research, In P. Hancock and N. Meshkati (Eds.), Human Mental Workload (pp. 139-183). Elsevier, Amsterdam, 1988.

Hinton, M. (2012, February 21). President Obama signs FAA bill into law. Unmanned Systems News. Retrieved from http://www.auvsi.org/AUVSI/News/\#FAAlaw.

Prevot, T. (2002). Exploring the Many Perspectives of Distributed Air Traffic Management: The Multi Aircraft Control System MACS. In: International Conference on Human-Computer Interaction in Aeronautics (pp. 23-25). 